\author{
Matthieu Resche-Rigon \\ Daniel Talmor \\ John P. Kress
}

\title{
Old wine in new bottles: should we publish old data?
}

Received: 6 November 2013

Accepted: 7 November 2013

Published online: 26 November 2013

(C) Springer-Verlag Berlin Heidelberg and ESICM 2013

M. Resche-Rigon (

UMR-S U717, INSERM, Paris, France

e-mail: matthieu.resche-rigon@univ-paris-diderot.fr

M. Resche-Rigon

Université Paris Diderot, Sorbonne Paris Cité, Paris, France

M. Resche-Rigon

SBIM, Hôpital Saint-Louis, APHP, Paris, France

D. Talmor

Department of Anesthesia, Critical Care and Pain Medicine, Beth Israel Deaconess Medical Center, Harvard Medical School, Boston, USA

\section{J. P. Kress}

Section of Pulmonary and Critical Care, Department of Medicine, University of Chicago, Chicago, USA

Clinical investigation in the intensive care unit (ICU) is difficult. Patients present with acute and rapidly changing conditions. Patients and their families are placed in an unfamiliar situation (i.e., the ICU) where vital decisions must be made rapidly. Obtaining informed consent in such an environment can be very challenging. For these reasons and a myriad of others, the conduct and timely completion of multicentered clinical trials in the ICU is a Herculean challenge. In this issue of Intensive Care Medicine, Lellouche and colleagues present data from a prospective randomized multicenter trial comparing noninvasive ventilation (NIPPV) using a heat and moisture exchanger (HME) compared to NIPPV using a heated humidifier $(\mathrm{HH})$ [1]. Contrary to recommendations published in 2012 that propose HH for NIPPV rather than HME, the authors found no difference in outcomes. This included a finding of similar intubation rates with the two humidification systems [1].

Upon reading this paper, a reader might ask, why a trial that finished enrolling patients in December 2003 would be published in 2013? Is it scientifically and ethically acceptable to publish a study after such a long delay? This question should be addressed in the context of the welldescribed phenomenon that many clinical trials are never published at all. Publication bias, whereby negative trials are less likely to be reported and published, is an important and well-described phenomenon [2]. Other reasons include poor study methodology and evaluation of a question that is no longer relevant.

In an era of rapidly advancing knowledge and technology, a study reporting data from a decade prior must be considered with particular scrutiny. There are several potential reasons for the delay in publication noted in this trial. During the review process, the authors acknowledged differences of opinion among themselves regarding interpretation of their results, with multiple analyses performed by different coauthors. Complex and conflicting schedules with regard to other research, clinical, educational, and administrative responsibilities may also have led to delays. Indeed, slow movement of clinical research projects involving centers from multiple cities or countries is not uncommon. For example, the results of the TracMan trial were originally presented at the 2009 Critical Care Canada meeting but not published until 2013 [3]. A decade between completing enrollment and submitting for publication would still seem excessively long even when taking the above issues into account.

One may argue that the considerations involved in publishing a study are the same regardless of age: is the study of high quality from a scientific and methodological point of review? The question can be rephrased as should we publish good old studies?

From a clinical point of view it is far from obvious in this case. Since 2003, evidence may have overtaken the 
question: another randomized clinical trial (RCT) could have been published or progress in large cohort studies using causal inference methodologies (such as propensity score) could have sufficiently advanced our knowledge on the topic. Moreover, the question could now be obsolete because of the emergence of new treatments or techniques. Importantly, this does not seem to be the case for this particular work on the impact of the use of heat and moisture exchangers or heat humidifiers during noninvasive ventilation [4-6]. Finally even if the trial is still relevant, one must question the external validity of the results. Rothwell has listed criteria for assessing the external validity of RCTs [7, 8]. Among them characteristics of randomized patients and differences between trial protocol and routine practice are of the highest importance. Are our patients and our routine practices the same 10 years later? We expect not. So, the usefulness of the results reported by Lellouche and colleagues needs to be put into perspective with regard to the change and improvement of the routine care that we now give to patients.

And yet, not publishing the results of this trial could be the lesser option. These data could still contribute to the total weight of the evidence on that topic via large collaborative systematic reviews or meta-analyses. One of the major concerns of such reviews is the publication bias and the subsequent overestimation of effects when "negative" studies face delayed publication [9-11]. Then, publications of data of good quality, albeit late results, are important and would improve precision of effect estimates. As in all science, medical science is cumulative; therefore reporting research results should be a moral obligation for all researchers [12]. This moral obligation includes several aspects. First there is a moral obligation towards other researchers who should be able to review all relevant evidence before beginning new research [13]. Secondly towards the community who pay for our research and have the right to demand that physicians have access to all the knowledge before treating a patient. This demand has led to considerable efforts to register RCTs and to maintain registries of active investigations. Finally there is a moral obligation towards the patients enrolled in the trial. These patients altruistically exposed themselves to unknown risk in order to participate in the trial and in the advancement of medical science. To have exposed them to that risk for nothing is to breach the moral contract that the investigators entered into with these subjects [13].

In summary, investigators have a responsibility to themselves, their subjects, and to the community at large for timely publication of the results of their work. Timely publication is important; however, in cases where outside circumstances have intervened and things are delayed, every reasonable attempt should still be made publish the data. In the future one may envision a role for both funding agencies as well as for study registries such as ClinicalTrials.gov in ensuring timely publication.

Conflicts of interest On behalf of all authors, the corresponding author states that there is no conflict of interest.

\section{References}

1. Lellouche F, L'Her E, Abroug F et al (2013) Impact of the humidification device on intubation rate during noninvasive ventilation with ICU ventilators: results of a multicenter randomized controlled trial. Intensive Care Med. doi:10.1007/s00134013-3145-z

2. Hopewell S, Loudon K, Clarke MJ et al (1996) Publication bias in clinical trials due to statistical significance or direction of trial results. Cochrane Database Syst Rev. doi:10.1002/ 14651858.MR000006.pub3

3. Young D, Harrison DA, Cuthbertson BH et al (2013) Effect of early vs late tracheostomy placement on survival in patients receiving mechanical ventilation: the tracman randomized trial. JAMA 309:2121-2129. doi:10. 1001/jama.2013.5154

4. Azoulay E, Kouatchet A, Jaber S et al (2013) Noninvasive mechanical ventilation in patients having declined tracheal intubation. Intensive Care Med 39:292-301. doi:10.1007/s00134-0122746-2
5. Bein T, Weber-Carstens S, Goldmann A et al (2013) Lower tidal volume strategy $(\approx 3 \mathrm{ml} / \mathrm{kg})$ combined with extracorporeal $\mathrm{CO} 2$ removal versus "conventional" protective ventilation $(6 \mathrm{ml} / \mathrm{kg})$ in severe ARDS: the prospective randomized Xtravent-study. Intensive Care Med 39:847-856. doi: 10.1007/s00134-012-2787-6

6. Vaschetto R, Turucz E, Dellapiazza F et al (2012) Noninvasive ventilation after early extubation in patients recovering from hypoxemic acute respiratory failure: a single-centre feasibility study. Intensive Care Med 38:1599-1606. doi:10.1007/ s00134-012-2652-7

7. Rothwell PM (2005) External validity of randomised controlled trials: "to whom do the results of this trial apply?" Lancet 365:82-93. doi:10.1016/S01406736(04)17670-8

8. Rothwell PM (2006) Factors that can affect the external validity of randomised controlled trials. PLoS Clin Trials. doi:10.1371/journal.pctr. 0010009
9. Guyatt GH, Oxman AD, Montori V et al (2011) GRADE guidelines: 5. Rating the quality of evidence-publication bias. J Clin Epidemiol 64:1277-1282. doi:10.1016/j.jclinepi.2011.01.011

10. Stern JM, Simes RJ (1997) Publication bias: evidence of delayed publication in a cohort study of clinical research projects. BMJ 315:640-645. doi: 10.1136/bmj.315.7109.640

11. Latronico N, Metelli M, Turin M et al (2013) Quality of reporting of randomized controlled trials published in intensive care medicine from 2001 to 2010. Intensive Care Med 39:1386-1395. doi:10.1007/s00134013-2947-3

12. Moher D (2007) Reporting research results: a moral obligation for all researchers. Can J Anaesth J Can Anesth 54:331-335. doi:10.1007/ BF03022653

13. Antes G, Chalmers I (2003) Underreporting of clinical trials is unethical. Lancet 361:978-979. doi:10.1016/ S0140-6736(03)12838-3 